\title{
CHARACTERIZING THE ROLE OF ORBITOFRONTAL CORTEX IN SOCIAL MEMORY
}

\author{
Marissa L Bruce ${ }^{1}$, Katharine D Andrews ${ }^{2}$, Elizabeth A Lungwitz ${ }^{2}$, William A \\ Truitt ${ }^{2,3}$
}

${ }^{1}$ Indiana Medical Student Program for Research and Scholarship ${ }^{2}$ Paul and Carole Stark Neurosciences Research Institute, ${ }^{3}$ Deptartment of Anatomy and Cell Biology, Indiana University School of Medicine, Indianapolis, IN, USA

\section{Background and Hypothesis:}

Social-enhanced safety learning is a psychosocial process used to reduce fear or anxiety by learning to discriminate fearful versus safe stimuli via a social safety cue. Learning to associate safety with a social cue requires intact social memory. Preliminary data in rats suggests inhibiting the orbitofrontal cortex (OFC) with pharmacologic agents impairs social memory. However, the specific mechanism by which OFC regulates social memory remains unknown. Because the OFC has broad functional implications including valuation, decision-making, social and emotional behaviors, olfaction, and non-social memory, we hypothesized that OFC inhibition was disrupting one of these specific processes, resulting in social memory impairment.

\section{Experimental Design or Project Methods:}

Cannulated adult male Sprague-Dawley rats were injected bilaterally in OFC with either saline vehicle, or $0.9 \mathrm{mM}$ Muscimol, a GABA agonist that transiently inhibits local neuronal activity. At 10 minutes post-injection, rats underwent behavior testing for either: social recognition, novel object recognition, social preference (innate gregariousness), or olfactory discrimination.

\section{Results:}

Rats receiving Muscimol injection, but not rats receiving vehicle injection, demonstrated statistically significant impairment of social recognition, observed as a failure to discriminate between two conspecifics. Alternatively, rats receiving Muscimol injection, but not rats receiving vehicle injection, did not demonstrate statistically significant impairment of novel object (non-social) recognition, innate gregariousness, or olfaction, which were all intact in vehicle injected rats.

\section{Conclusion and Potential Impact:}

These data suggest OFC may be part of a unique neural circuit specific to social memory. Delineating the circuitry of social memory from non-social memory offers exciting possibilities in the advancement of precision therapies. 